Research Paper

\title{
The value of preoperative Glasgow Prognostic Score and the C-Reactive Protein to Albumin Ratio as prognostic factors for long-term survival in pathological T1N0 esophageal squamous cell carcinoma
}

\author{
Xiangyang $\mathrm{Yu}^{1,2,{ }^{*}}$, Yingsheng Wen ${ }^{1,2,}{ }^{*}$, Yongbin $\operatorname{Lin}^{1,2}$, Xuewen Zhang ${ }^{1,3}$, Yongqiang Chen ${ }^{1,2}$, Weidong \\ Wang1,2, Gongming Wang1,2, Lanjun Zhang1,2凶 \\ 1. State Key Laboratory of Oncology in South China, Collaborative Innovation Center for cancer medicine. \\ 2. Department of Thoracic Surgery, Sun Yat-sen university Cancer center, 651 Dong Feng East Road, Guangdong, China. \\ 3. Department of Medical Oncology, Sun Yat-sen University Cancer Center, 651 Dong Feng East Road, Guangzhou, China. \\ *Xiangyang Yu and Yingsheng Wen contributed equally to this work. \\ $\square$ Corresponding author: Dr. Lanjun Zhang, M.D., Ph.D, Director Office of Thoracic Surgery, 651 Dong Feng East Road, Guangzhou, 510060, China. E-mail: \\ zhanglj@sysucc.org.cn. \\ (C) Ivyspring International Publisher. This is an open access article distributed under the terms of the Creative Commons Attribution (CC BY-NC) license \\ (https://creativecommons.org/licenses/by-nc/4.0/). See http://ivyspring.com/terms for full terms and conditions.
}

Received: 2017.09.08; Accepted: 2017.12.04; Published: 2018.02.12

\begin{abstract}
Plenty of studies have confirmed the prognostic values of inflammation-based prognostic scores in many malignant tumors. In present study, we aim to explore whether these indexes has same prognostic values in patients with stage TINO esophageal squamous cell carcinoma (ESCC). The clinicopathological data of 160 consecutive patients with pathological stage TINO ESCC from January 2005 to December 2012 were collected retrospectively. As prognostic factors, the inflammation-based prognostic scores, including C-reactive protein (CRP), Glasgow prognostic score (GPS), prognostic index (PI), neutrophil to lymphocyte ratio (NLR), platelet to lymphocyte ratio (PLR) and CRP to albumin ratio (CAR), were evaluated. The best cut-off values were determined by the receiver operating characteristic (ROC) curves. The median follow-up time was 71.8 months. During the follow-up period, $34(21.3 \%)$ patients occurred postoperative recurrence and $30(18.8 \%)$ tumor-related deaths were recorded. The best cut-off values of CRP, NLR, PLR and CAR were 1.090, 1.976, 103.200 and 0.023 , respectively. After multivariate analysis, the GPS and CAR were identified as independently prognostic factors for overall survival (OS) $(p=0.017$ and 0.040 , respectively). Of all 160 individuals, there were $86(53.8 \%)$ and $85(53.1 \%)$ patients classified into high GPS group $(1-2)$ and elevated CAR group $(>0.023)$, respectively. In addition, the GPS were positively associated with PI $(p<0.000)$ and the levels of serum CRP $(p<0.000), N L R(p=0.004), P L R$ $(p=0.029)$ and CAR $(p<0.000)$ and the above correlations were also observed between the CAR and other inflammation-based prognostic scores (all $p<0.050$, except for $p=0.054$ for PLR levels). The preoperative GPS and CAR were simple, inexpensive, readily available predictor for long-term survival in stage TINO ESCC patients who underwent esophagectomy.
\end{abstract}

Key words: early esophageal cancer, prognostic factors, inflammation, esophagectomy

\section{Introduction}

Esophageal cancer (EC) is high incidence and mortality not just in China, which was reported as the fourth most commonly diagnosed malignant tumor and also the fourth most common cause of cancer-related death in 2015, but also in some Western and Central Asian countries [1, 2]. Notably, almost $70 \%$ of the initial visits of patients with EC were diagnosed as advanced stages (regional or distant 
metastasis) and the five-year overall survival (OS) of this population was range from $10 \%$ to $31 \%$ even after multidisciplinary therapies [3-5]. In the past decades, with the cancer screening promoted by Chinese anti-cancer association in high incidence area of EC, more and more patients with early stage tumors are detected [1]. In spite of the ability to detect and resect these early stage tumors, but still 10\%-35.9\% of these patients would occur tumor-related death within five years after radical resection [4, 6]. Therefore, identifying independent prognostic factors for superficial EC (T1) is a very meaningful topic. At the same time, these risk factors could not only provide a reasonable choice in endoscopic resection (ER) or esophagectomy, but may also instruct postoperative adjuvant treatment.

Inflammation-based prognostic scores have intensively been studied in variety of malignant solid tumors and are emerging as promising prognostic indexes. However, no studies have explored the prognostic value of the above mentioned scores in patients with superficial esophageal squamous cell carcinoma (ESCC). In addition, patients with stage T1 ESCC are candidates for ER, which is recommend by guidelines from Chinese Society of Clinical Oncology (CSCO), Japan Esophageal Society (JES) and National Comprehensive Cancer Network (NCCN) etc, but the accurate prognostic markers to identify the high-risk patients who may easily recurrence after ER are essential. Furthermore, due to the majority stage T1N0 patients with ESCC owning long-term survival, only length and depth of tumor invasion, microscopic tumor budding, poor differentiation and lymphovascular invasion had been found as independent prognostic indicators for postoperative OS reported by limited literatures [7-10]. Therefore, establishing other simple, inexpensive, and promising prognostic factors for superficial ESCC (T1) may seem difficult but necessary. What's more, the preoperative peripheral blood examinations are routinely preformed and readily available.

Thus, this study is focus on pathological T1N0 Chinese patients with the common pathological subtype-squamous cell carcinoma-to explore the prognostic values of inflammation-based prognostic scores.

\section{Materials and Methods}

\section{Patient selection and Ethics statement}

1994 consecutive patients with ESCC underwent esophagectomy at department of thoracic surgery, Sun Yat-sen university cancer center (SYSUCC) between January 2005 and December 2012 were enrolled in initial database. And all clinical, pathological, radiological, preoperative inflammation-based indicators, therapeutic strategy as well as follow-up information were retrospectively referred and typed into statistical software simultaneously. Of course, the written informed consents were signed by patients themselves and the hospital ethics committee in SYSUCC had approved this study based on the World Medical Association Declaration of Helsinki preparatory to our all work.

Patients were enrolled in final analysis if they met following criteria: (1) there was no neoadjuvant treatment; (2) no distant metastasis was found by preoperative radiological scanning; (3) all of the patients were given complete gross tumor resection with safety margins (R0 resection), which is defined as no microscopic involvement in the surgically resected margins, and 2- or 3-field lymph node dissection; (3) postoperative diagnosis of T1 stage ESCC (the lamina propria, muscularis mucosae or submucosa invasion) had been established by two board-certified pathologist independently and all lymph node was confirmed negative (N0).

Patients were excluded based on following criteria: (1) accompany with autoimmune disease or preoperative infection; (2)other malignant neoplas$\mathrm{m}(\mathrm{s})$ was detected before or after esophagectomy; (3) death during perioperative period; (4) without preoperative data of peripheral blood cells count; (5) loss to follow-up.

\section{Definitions of inflammation-based prognostic scores}

As previously described [11], the Glasgow prognostic score (GPS) is simultaneously consisted by nutritional (if albumin $\geq 3.5 \mathrm{mg} / \mathrm{dl}$ was allocated a score of 0 , otherwise 1 ) and inflammatory conditions (if $\mathrm{CRP} \leq 1.0 \mathrm{mg} / \mathrm{dl}$ was allocated a score of 0 , otherwise 1). In term of modified GPS (mGPS), both an abnormal CRP (>1.0 mg/l) and hypoalbuminaemia $(<3.5 \mathrm{mg} / \mathrm{dl})$ were allocated a score of 2 , and only one or none of the two biochemical abnormalities was allocated a score of 1 or 0 , respectively. In addition, except the cut-off value of CRP (abnormality: $>0.3$ $\mathrm{mg} / \mathrm{l})$, the allocation method of high-sensitivity modified GPS (HS-mGPS) was the same as the mGPS.

Differ to GPS, the prognostic index (PI) consists of two inflammatory parameters: CRP (if $\leq 1.0 \mathrm{mg} / \mathrm{dl}$ was allocated a score of 0 , otherwise 1 ) and white cell count (WBC, if $\leq 11,000 / \mu$ was allocated a score of 0 , otherwise 1). After summation of respective allocated score, these patients were divided into three groups, including 0,1 and 2 .

Other prognostic indicators are calculated by following mathematical formula: (1) neutrophil to lymphocyte ratio $(\mathrm{NLR})=$ total neutrophil count / 
total lymphocyte count; (2) platelet to lymphocyte ratio $(\mathrm{PLR})=$ total platelet count $/$ total lymphocyte count; (3) CRP to albumin ratio $(\mathrm{CAR})=\mathrm{CRP}(\mathrm{mg} / \mathrm{L})$ / albumin (g/L).

\section{Follow-up strategy and statistical analysis}

The follow-up observation was performed since the date of radical resection and the interval of every follow-up visit were every three months during the first two years, every six months since the beginning of the third year to the end of the fifth year, and then each subsequent annual. Each follow-up protocol routinely consisted of complete blood count, blood biochemical examination, common tumor markers, esophageal barium meal, head and neck ultrasonography, as well as upper abdominal ultrasonography. In addition, the thoracoabdominal contrast-enhanced CT scanning was carried out once every six months during the first three years and then once a year until death occurred in our facility. The time of disease-free survival (DFS) was calculated from the date of esophagectomy to the first local recurrence or distant metastasis observed by radiology findings. Similarly, the time interval from the date of esophagectomy to death caused by ESCC was defined as the total survival time.

All statistical analyses were performed by SPSS 23.0 (SPSS Inc., Chicago, IL, USA) and the two-sided $p$ value less than 0.050 was defined as significantly difference in statistics, but the survival curves were constructed using GraphPad Prism 7.0 (GraphPad Software Inc., La Jolla, USA). Firstly, the best cut-off values of these inflammation-based prognostic scores were determined by maximum Youden index, which were generated by respective receiver operating characteristics (ROC) curves, to evaluate the predictive values in prognosis. In addition, the differences of categorical variables between groups were studied using chi-square test. Needless to say, the prognostic factors of DFS and overall survival (OS) were confirmed by Kaplan-Meier univariate analysis using log-rank test. And then these significantly prognostic variables $(p<0.050)$ obtained from Kaplan-Meier analysis were enrolled in Cox multivariate regression analysis based on forward stepwise method.

\section{Results}

\section{Clinical, pathological and surgical characteristics}

There were 160 consecutive patients with ESCC diagnosed as pathological stage T1N0 enrolled, including 105 male and 55 female (median age: 59 years; interquartile range: 52 to 65 years). More than half of these patients $(53.75 \%, 86$ of 160$)$ had smoked but a history of alcohol use was recorded only in 22 patients $(13.75 \%)$. Of the 42 patients with family history of malignant tumors, 30 patients came from esophageal cancer family. The majority of these tumors $(65.00 \%, 104$ of 160$)$ located in middle segment of the thoracic esophagus. In addition, the Sweet (transthoracic esophagectomy with two-field lymphadenectomy) and McKeown (tri-incisional esophagectomy with three-field lymphadenectomy) surgical approach were performed in 102 patients and 57 patients, respectively. The median and average count of examined lymph nodes (LNs) was 17.0 and 19.9, respectively (interquartile range: 12 to 25), and the median and average stations of examined LNs was 5.0 and 6.2, respectively (interquartile range: 4 to $7)$. In our cohort, only six individuals received postoperative paclitaxel plus cisplatin or nedaplatin regimen for adjuvant chemotherapy because of poorly differentiation. All the baseline characteristics are described in Table 1.

\section{Best cut-off values}

Calculated by the above mathematical formulas, the values of NLR, PLR, CAR range from 0.460-14.000 (median: 1.895), 41.540-597.140 (median: 102.364), 0.000-1.040 (median: 0.025), respectively. Based on the maximum Youden index, 1.976, 103.200, 0.023 were chosen as the best cut-off nodes of NLR, PLR, CAR for OS, respectively (Fig. 1).

\section{Prognostic factors for DFS and OS}

The follow-up data were updated to 1 June 2017 and the median follow-up time was 71.8 months (interquartile range: 52.0 to 95.5 months). During the follow-up period, there were only 34 patients occurred postoperative recurrence of ESCC including 13 distant organ metastasis and 21 local recurrences, and the 1-, 3-, 5- and 10-year DFS rate were 96.2\%, $85.6 \%, 78.7 \%$ and $74.7 \%$, respectively. In addition, 30 cases of all 42 deaths were directly related to recurrence of ESCC, and the 1-, 3-, 5- and 10-year OS rate were $94.4 \%, 82.2 \%, 79.4 \%$ and $66.3 \%$, respectively.

In univariate analysis, the PI 0, GPS 0 , and low CRP $(\leq 1.090 \mathrm{mg} / \mathrm{l}), \mathrm{NLR}(\leq 1.976), \mathrm{PLR}(\leq 103.200)$, CAR $(\leq 0.023)$ were significantly correlated with longer DFS and OS (all p<0.050, Table 1). In addition, these patients with a history of alcohol consumption showed significantly shorter 10-year DFS and OS (DFS: $58.4 \%$ vs. $77.3 \%$, p=0.015; OS: $59.1 \%$ vs. $58.1 \%$, $\mathrm{p}=0.040)$. The best cut point of the resected lymph nodes count (RLNs) was determined as 13 in our study based on ROC curve and we had found that the 10-year DFS was significantly longer for RLNs $>13$ than for RLNs $\leq 13$ (79.2\% vs. $65.3 \%$, p=0.048), but not in OS ( $\mathrm{p}=0.061)$. 
Table 1. Univariate analysis for disease-free survival (DFS) and overall survival (OS) in 160 patients with pathological T1N0 esophageal squamous cell carcinoma

\begin{tabular}{|c|c|c|c|c|c|}
\hline \multirow[t]{2}{*}{ Variables } & \multirow{2}{*}{$\begin{array}{l}\text { No. of patients } \\
(N=160)\end{array}$} & \multicolumn{4}{|c|}{ Univariate analysis } \\
\hline & & 10-year DFS & $\mathbf{P}$ & 10-year OS & $\mathbf{P}$ \\
\hline \multicolumn{6}{|l|}{ Gender } \\
\hline Male & 105 & $77.0 \%$ & 0.356 & $54.3 \%$ & 0.823 \\
\hline Female & 55 & $82.1 \%$ & & $71.0 \%$ & \\
\hline \multicolumn{6}{|c|}{ Age (years, mean \pm SD) } \\
\hline$<65$ & 119 & $77.9 \%$ & 0.315 & $63.9 \%$ & $0.008^{*}$ \\
\hline$\geq 65$ & 41 & $60.5 \%$ & & $42.1 \%$ & \\
\hline \multicolumn{6}{|c|}{ Smoking history } \\
\hline Yes & 86 & $76.9 \%$ & 0.469 & $74.1 \%$ & 0.462 \\
\hline No & 74 & $72.3 \%$ & & $50.1 \%$ & \\
\hline \multicolumn{6}{|c|}{ Alcohol consumption } \\
\hline Yes & 22 & $58.4 \%$ & $0.015^{*}$ & $59.1 \%$ & $0.040^{*}$ \\
\hline No & 138 & $77.3 \%$ & & $58.1 \%$ & \\
\hline \multicolumn{6}{|c|}{ Family malignant tumor history } \\
\hline Yes & 42 & $77.4 \%$ & 0.200 & $74.5 \%$ & 0.240 \\
\hline No & 118 & $68.3 \%$ & & $53.0 \%$ & \\
\hline \multicolumn{6}{|c|}{ Preoperative loss of weight } \\
\hline$<3$ kilograms & 115 & $76.0 \%$ & 0.228 & $52.9 \%$ & 0.206 \\
\hline$\geq 3$ kilograms & 45 & $71.2 \%$ & & $64.6 \%$ & \\
\hline \multicolumn{6}{|c|}{ Carcinoma differentiation } \\
\hline Highly & 24 & $69.8 \%$ & 0.925 & $59.3 \%$ & 0.939 \\
\hline Moderately & 84 & $75.3 \%$ & & $54.4 \%$ & \\
\hline Poorly & 52 & $77.0 \%$ & & $69.7 \%$ & \\
\hline \multicolumn{6}{|c|}{ Tumor location } \\
\hline Upper & 26 & $81.1 \%$ & 0.708 & $84.3 \%$ & 0.191 \\
\hline Middle & 104 & $76.2 \%$ & & $55.2 \%$ & \\
\hline Lower & 30 & $62.9 \%$ & & $58.9 \%$ & \\
\hline \multicolumn{6}{|c|}{ Length of tumor } \\
\hline$\leq 1.8 \mathrm{~cm}$ & 42 & $72.4 \%$ & 0.650 & $39.4 \%$ & 0.105 \\
\hline$>1.8 \mathrm{~cm}$ & 118 & $75.4 \%$ & & $72.6 \%$ & \\
\hline \multicolumn{6}{|c|}{ Surgical approach } \\
\hline Sweet & 102 & $75.8 \%$ & 0.980 & $55.1 \%$ & 0.926 \\
\hline Mckeown & 57 & $70.6 \%$ & & $68.5 \%$ & \\
\hline \multicolumn{6}{|c|}{ Adjuvant therapy } \\
\hline Yes & 6 & $66.7 \%$ & 0.605 & $100 \%$ & 0.168 \\
\hline No & 154 & $75.2 \%$ & & $57.0 \%$ & \\
\hline Resected lymp & & & & & \\
\hline$\leq 13$ & 53 & $65.3 \%$ & $0.048^{*}$ & $44.7 \%$ & 0.061 \\
\hline$>13$ & 107 & $79.2 \%$ & & $74.1 \%$ & \\
\hline Prognostic ind & & & & & \\
\hline 0 & 74 & $87.2 \%$ & $0.003^{*}$ & $81.7 \%$ & $0.004^{*}$ \\
\hline 1 & 80 & $59.2 \%$ & & $26.4 \%$ & \\
\hline 2 & 6 & $100 \%$ & & $55.6 \%$ & \\
\hline Glasgow prog & & & & & \\
\hline 0 & 74 & $87.2 \%$ & $0.004^{*}$ & $81.7 \%$ & $0.000^{*}$ \\
\hline $1-2$ & 86 & $61.9 \%$ & & $26.5 \%$ & \\
\hline Modified GPS & & & & & \\
\hline 0 & 74 & $87.2 \%$ & $0.004^{*}$ & $81.7 \%$ & $0.000^{*}$ \\
\hline $1-2$ & 86 & $61.9 \%$ & & $26.5 \%$ & \\
\hline High-sensitivi & & & & & \\
\hline 0 & 15 & $86.2 \%$ & 0.328 & $93.3 \%$ & 0.062 \\
\hline $1-2$ & 145 & $73.2 \%$ & & $53.9 \%$ & \\
\hline$C$ reactive prot & & & & & \\
\hline$\leq 1.090$ & 80 & $84.0 \%$ & $0.019^{*}$ & $81.7 \%$ & $0.000^{*}$ \\
\hline$>1.090$ & 80 & $63.5 \%$ & & $25.7 \%$ & \\
\hline Neutrophil to & & & & & \\
\hline$\leq 1.976$ & 88 & $81.6 \%$ & $0.032^{*}$ & $65.8 \%$ & $0.002^{*}$ \\
\hline$>1.976$ & 72 & $65.3 \%$ & & $50.6 \%$ & \\
\hline Platelet to lym & & & & & \\
\hline$\leq 103.200$ & 83 & $83.3 \%$ & $0.047^{*}$ & $64.3 \%$ & $0.011^{*}$ \\
\hline$>103.200$ & 77 & $65.2 \%$ & & $51.1 \%$ & \\
\hline CRP to album & & & & & \\
\hline$\leq 0.023$ & 75 & $84.4 \%$ & $0.021^{*}$ & $83.5 \%$ & $0.000^{*}$ \\
\hline$>0.023$ & 85 & $63.7 \%$ & & $25.4 \%$ & \\
\hline
\end{tabular}

No., number; DFS, disease-free survival; OS, overall survival; SD, standard deviation.

*the $\mathrm{p}$ value was considered as significantly difference in statistic because of less than 0.050 . 


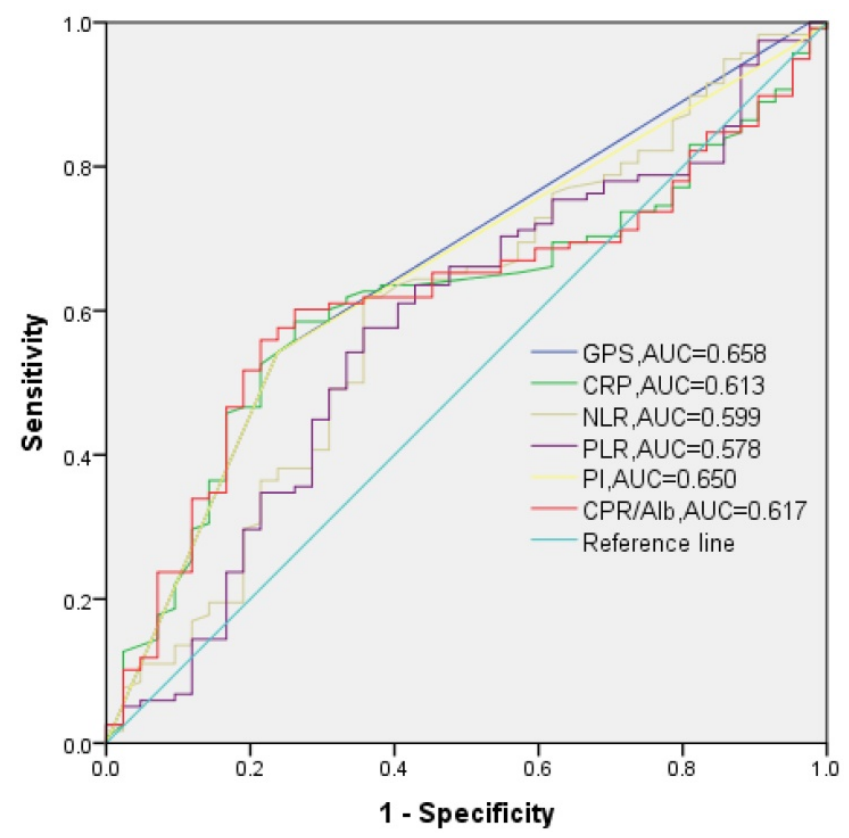

Figure 1. The receiver operating characteristic (ROC) curves of the GPS (categorical), PI (categorical), CRP (continuous), NLR (continuous), PLR (continuous) and CAR (continuous) to determinate the best cut-off value at 10 years of follow-up.

After multivariate analysis, there was no clinicopathological, inflammatory or surgical factors indicated as prognostic index for DFS (all $\mathrm{p}>0.050$ ). However, the GPS 0 (HR: 0.068, 95\% CI: 0.007-0.622, $\mathrm{P}=0.017$ ) and low CAR ( $\leq 0.023$, HR: 0.126, 95\% CI: 0.017-0.911, $\mathrm{P}=0.040$, Table 2 and Fig. 2) were predictive of better long-term survival in patients with pathological T1N0 ESCC.

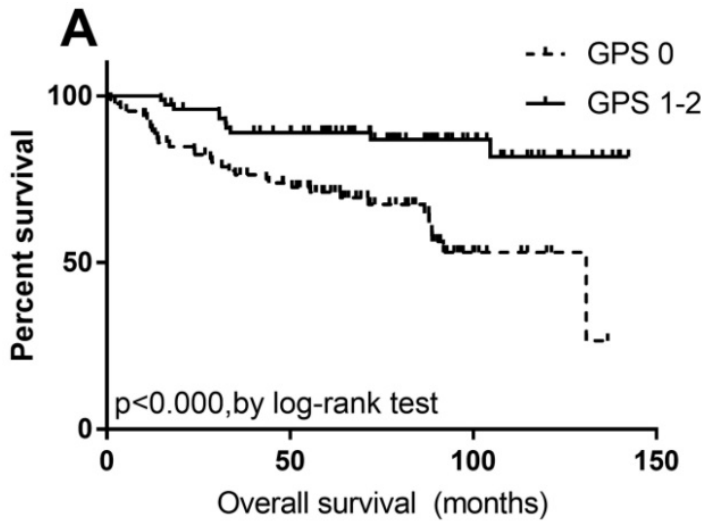

\section{Relationships between inflammation-based prognostic scores and clinicopathological variables}

The relationships between GPS, CAR and clinicopathological variables were described in Table 3. Only the distribution of age was found statistically difference between the elevated and low CAR groups $(\leq 0.023 />0.023, \mathrm{p}=0.014)$. The population distribution based on PI score (0/1-2) was completely consistent with the GPS (0/1-2). Similarly, the majority of these patients who were set into low CAR group $(\leq 0.023)$ were also set into PI score 0 and GPS 0 group (72 of 75, $96.0 \%)$. In addition, the correlation analysis indicated that the two GPS (0/1-2) groups were positively associated with the levels of serum CRP, NLR, PLR and CAR (all p<0.050). And the above correlations were also observed between the two CAR groups $(\leq 0.023 />0.023$, all $p<0.050$, except for $p=0.054$ for PLR levels).

Table 2. Multivariate analysis for overall survival (OS) in 160 patients with pathological TINO esophageal squamous cell carcinoma

\begin{tabular}{llll}
\hline Variables & Groups & Hazard ratio $(95 \%$ CI $)$ & P \\
\hline Age & $<65 / \geq 65$ & $0.661(0.332-1.313)$ & 0.237 \\
$\begin{array}{l}\text { Alcohol } \\
\text { consumption }\end{array}$ & Yes/No & $0.523(0.244-1.121)$ & 0.095 \\
CRP & $\leq 1.090 />1.090$ & $0.673(0.088-5.146)$ & 0.703 \\
PI & $0 / 1 / 2$ & $0.284(0.008-10.717)$ & 0.496 \\
GPS/mGPS & $0 / 1-2$ & $0.068(0.007-0.622)$ & $0.017^{*}$ \\
NLR & $\leq 1.976 />1.976$ & $0.641(0.308-1.336)$ & 0.235 \\
PLR & $\leq 103.200 />103.200$ & $0.580(0.288-1.166)$ & 0.126 \\
CAR & $\leq 0.023 />0.023$ & $0.126(0.017-0.911)$ & $0.040^{*}$ \\
\hline
\end{tabular}

CI, confidence interval, CRP, $C$ reactive protein, PI, prognostic index, GPS, Glasgow prognostic score, mGPS, modified Glasgow prognostic score, NLR, neutrophil to lymphocyte ratio, PLR, platelet to lymphocyte ratio, CAR, CRP to albumin ratio.

*the $\mathrm{p}$ value was considered as significantly difference in statistic because of less than 0.050 .

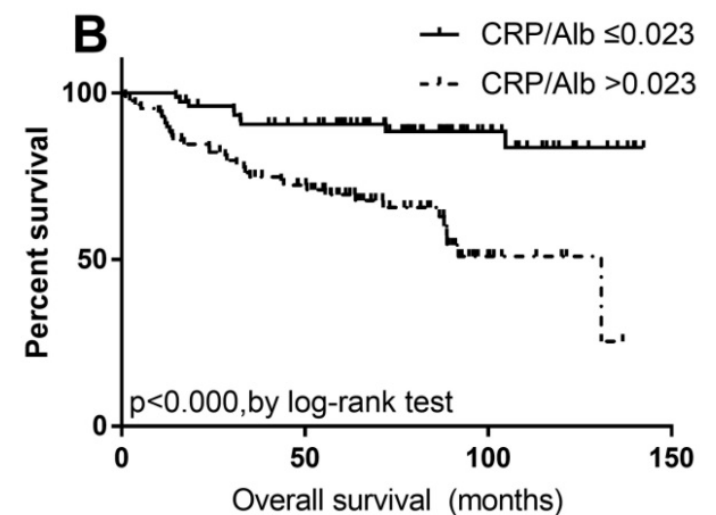

Figure 2. Kaplan-Meier analysis with the log-rank test for OS of 160 patients with pathological T1N0 esophageal squamous cell carcinoma, according to (A) GPS $(0 / 1-2)$ and $(B)$ CAR $(\leq 0.023 />0.023)$. 
Table 3. The correlation of GPS and CAR with the clinicopathological features in 160 patients with pathological T1N0 esophageal squamous cell carcinoma

\begin{tabular}{|c|c|c|c|c|c|c|}
\hline \multirow[t]{2}{*}{ Variables } & \multicolumn{3}{|l|}{ GPS } & \multicolumn{3}{|l|}{ CAR } \\
\hline & 0 & $1-2$ & $\mathbf{P}$ & $\leq 0.023$ & $>0.023$ & $\mathbf{P}$ \\
\hline Age (years, mean \pm SD) & $57.8 \pm 8.0$ & $59.7 \pm 9.0$ & 0.158 & $57.1 \pm 8.4$ & $60.4 \pm 8.5$ & $0.014^{*}$ \\
\hline $\begin{array}{l}\text { Preoperative loss of weight } \\
(\text { mean } \pm \text { SD) }\end{array}$ & $2.020 \pm 3.001$ & $1.994 \pm 4.070$ & 0.964 & $2.033 \pm 3.024$ & $1.982 \pm 4.067$ & 0.929 \\
\hline Length of tumor (mean \pm SD) & $2.340 \pm 1.000$ & $2.573 \pm 1.295$ & 0.210 & $2.324 \pm 1.001$ & $2.591 \pm 1.291$ & 0.150 \\
\hline \multicolumn{7}{|l|}{ Gender } \\
\hline Male & $49(66.2)$ & $56(65.1)$ & 0.884 & $51(68.0)$ & $54(63.5)$ & 0.552 \\
\hline Female & $25(33.8)$ & $30(34.9)$ & & $24(32.0)$ & $31(36.5)$ & \\
\hline \multicolumn{7}{|l|}{ Smoking history } \\
\hline Yes & $44(59.5)$ & $42(48.8)$ & 0.179 & $44(58.7)$ & $42(49.4)$ & 0.241 \\
\hline No & $30(40.5)$ & $44(51.2)$ & & $31(41.3)$ & $43(50.6)$ & \\
\hline \multicolumn{7}{|l|}{ Alcohol consumption } \\
\hline Yes & $7(9.5)$ & $15(17.4)$ & 0.171 & $8(10.7)$ & $14(16.5)$ & 0.287 \\
\hline No & $67(90.5)$ & $71(82.6)$ & & $67(89.3)$ & $71(83.5)$ & \\
\hline \multicolumn{7}{|l|}{$\begin{array}{l}\text { Family malignant } \\
\text { tumor history }\end{array}$} \\
\hline Yes & $21(28.4)$ & $21(24.4)$ & 0.570 & $23(30.7)$ & $19(22.4)$ & 0.233 \\
\hline No & $53(71.6)$ & $65(75.6)$ & & $52(69.3)$ & $66(77.6)$ & \\
\hline \multicolumn{7}{|l|}{ Carcinoma differentiation } \\
\hline Highly & $10(13.5)$ & $14(16.3)$ & 0.246 & $10(13.3)$ & $14(16.5)$ & 0.081 \\
\hline Moderately & $35(47.3)$ & $49(57.0)$ & & $34(45.4)$ & $50(58.8)$ & \\
\hline Poorly & $29(39.2)$ & $23(26.7)$ & & $31(41.3)$ & $21(24.7)$ & \\
\hline \multicolumn{7}{|l|}{ Tumor location } \\
\hline Upper & 14(18.9) & 12(14.0) & 0.425 & 14(18.7) & 12(14.3) & 0.392 \\
\hline Middle & $48(64.9)$ & $55(64.0)$ & & $50(66.7)$ & $53(63.1)$ & \\
\hline Lower & $11(14.9)$ & $19(22.0)$ & & 11(14.6) & $19(22.6)$ & \\
\hline \multicolumn{7}{|l|}{ Prognostic index } \\
\hline 0 & $74(100)$ & $0(0.0)$ & $0.000^{*}$ & $72(96.0)$ & $2(2.4)$ & $0.000^{*}$ \\
\hline $1-2$ & $0(0.0)$ & $86(100)$ & & $3(4.0)$ & $83(97.6)$ & \\
\hline \multicolumn{7}{|l|}{ Glasgow prognostic score(GPS) } \\
\hline 0 & - & - & - & $72(96.0)$ & $2(2.4)$ & $0.000^{*}$ \\
\hline $1-2$ & - & - & & $3(4.0)$ & $83(97.6)$ & \\
\hline $\begin{array}{l}C \text { reactive protein }(C R P, \mathrm{mg} / \mathrm{l}) \\
(\mathrm{mean} \pm \mathrm{SD})\end{array}$ & $0.534 \pm 0.249$ & $4.050 \pm 5.796$ & $0.000^{*}$ & $0.541 \pm 0.256$ & $4.085 \pm 5.822$ & $0.000^{*}$ \\
\hline $\begin{array}{l}\text { Neutrophil to lymphocyte ratio } \\
\text { (mean } \pm \text { SD) }\end{array}$ & $1.868 \pm 0.946$ & $2.536 \pm 1.740$ & $0.003^{*}$ & $1.871 \pm 0.966$ & $2.541 \pm 1.737$ & $0.004^{*}$ \\
\hline $\begin{array}{l}\text { Platelet to lymphocyte ratio } \\
\text { (mean } \pm \text { SD) }\end{array}$ & $103.493 \pm 41.821$ & $124.154 \pm 70.904$ & $0.029^{*}$ & $104.901 \pm 42.772$ & $123.154 \pm 70.990$ & 0.054 \\
\hline $\begin{array}{l}\text { CRP to albumin ratio(CAR) } \\
(\text { mean } \pm S D)\end{array}$ & $0.012 \pm 0.006$ & $0.098 \pm 0.151$ & $0.000^{*}$ & - & - & - \\
\hline
\end{tabular}

GPS, Glasgow prognostic score; CAR, CRP to albumin ratio; SD, standard deviation.

*the $\mathrm{p}$ value was considered as significantly difference in statistic because of less than 0.050 .

\section{Discussion}

Inflammation had been documented as a part of the normal but complex biological response to sterile cell death or foreign infection since the origination of available medical history, which could remove or neutralize injurious material and activate healing and regeneration. As time went on, the potential correlation between leukocytes invasion and tumors had been firstly observed by Rudolf Virchow in the mid-19th century. Since then, more and more mechanism studies have clarified that tumorassociated inflammatory response play a critical role in the initiation, promotion, malignant conversion, invasion and metastasis of varies malignant solid tumor development [12]. When the progression or metastasis of malignancies occurred, the inflammatory cytokines, such as interleukin 1 (IL-1), interleukin 6 (IL-6), chemokines, NF-kB etc, were secreted by tumor-infiltrating lymphocytes or tumor cells, which could promote the immunity and hematopoietic system, including neutrophils, lymphocytes, CRP, platelets etc. Thus the elevated levels or ratios of inflammatory cells could be predictive factors for recurrence after therapy and cancer-related death and the prognostic values for many malignancies had been elaborated elsewhere [13-16].

The elevated serum CRP level had been reported only once as a significant prognostic parameter for 5-year survival in early stage ESCC patients (T1-2N0) by Zheng-Bo Song et al [17]. However, in our retrospective analysis, the single parameter lost the prognostic ability for long-term survival in stage T1N0 ESCC patients. As previous elaboration, the serum CRP level could not only reflect host 
acute-phase inflammatory response, but also associated with the nutrition status. In other words, the malnutrition status could weaken systemic inflammatory response leading to low serum CRP level [18]. Thus the inflammatory response protein (such as CRP) combining with systemic nutrition status, especially albumin, may be more sensitive marker for prognosis. The Glasgow prognostic score (GPS), which could evaluate inflammatory and nutrition status simultaneously, was firstly reported as prognostic factor for inoperable non-small cell lung cancer by Forrest et al in 2003 and the prognostic value had been proved in early, advanced or non-selected stage cancer by many literature review and meta-analysis [15]. As previous studies, the patients who were classified into the group of score 2 were limited (only 1 case in this study) $[19,20]$. Xiao-li Wei et al, Xiao-ling Xu et al and G. Jomrich et al had reported that high GPS (1-2) was not significantly associated with shorter OS in non-selected or resectable ESCC patients, but, multivariate analysis identified that GPS (0/1-2) was the independent prognostic factor for 10-year OS among stage T1N0 ESCC in our study [20-22], which was in accordance with the result from N. Hirahara et al among elderly ESCC patients (age $\geq 70$ years) [19]. In 2007, the first study in colon and rectal cancer from McMillan DC et al indicated that the modified GPS (mGPS) was significantly associated with overall survival [23]. However, the novel mGPS in predicting survival for ESCC still needs to be verified in selected or large cohort and the value has not been established by previous studies $[20,21,24]$. In this study, the population distribution based on mGPS $(0 / 1 / 2)$ was completely consistent with the GPS $(0 / 1 / 2)$ and thus the prognostic value was still unclear. The summary of these relevant studies are showed in supplementary table (Table S1) [25-27].

In 2015, a novel inflammation-based prognostic score - the CRP/Alb ratio (CAR) - was introduced by Kinoshita et al in non-selected hepatocellular carcinoma patients and they had proved that its prognostic ability could be comparable to mGPS and better than NLR [28]. In the same year, Xiao-ling Xu et al and Xiao-li Wei et al had explored the prognostic value of CAR in operable and non-selected ESCC patients, respectively and the area under the curve (AUC) values of the CAR was proved higher than the other inflammation-based prognostic scores at 12 months, 24 months, and 36 months [20, 21], which was consistent with our analysis [21]. In addition, similar to the above mentioned reports, the novel but promising CAR has been identified as an independent prognostic factor for OS in our study by multivariate analysis as well $[29,30]$. The summary of these relevant studies are showed in supplementary table (Table S2).

In our study, we had found that the elevated CAR was only connected with elderly patients $(p=0.014)$, but no significant correlation was observed between GPS (0/1-2) and clinicopathological features, which was not in agreement with the previous findings in ESCC [19-21, 31]. It is probably because the tumor stage was specific T1N0 in our cohort, but non-selected in previous literatures. What's more, Pearson's correlation indicated that the GPS and CAR level were significantly associated with the PI score $(\mathrm{r}=0.931, \mathrm{p}<0.000$ and $\mathrm{r}=0.356, \mathrm{p}<0.000$; respectively $)$ and the levels of serum CRP $(r=0.432, p<0.000$ and $\mathrm{r}=0.989, \mathrm{p}<0.000$; respectively), NLR $(\mathrm{r}=0.218, \mathrm{p}=0.006$ and $\mathrm{r}=0.183, \mathrm{p}=0.021$; respectively), PLR $(\mathrm{r}=0.165$, $\mathrm{p}=0.037$ and $\mathrm{r}=0.082, \mathrm{p}=0.300$; respectively). The two indexes may absorb the prognostic values of all other inflammation-based prognostic scores and show a combined prognostic ability. In addition, the AUC was 0.658 for GPS (95\% CI: 0.564-0.752, $\mathrm{p}=0.002)$ and 0.617 for CAR (95\% CI: 0.525-0.709, p=0.025, Fig. 1). Thus, to stage T1N0 ESCC patients who underwent esophagectomy, we recommend the preoperative GPS and CAR as predicative markers for OS and the predicative value of GPS, which could be well standardized, was slightly superior to the CAR.

A recent meta-analysis, which included seven retrospective, observational, cohort studies involving 1540 patients with EC, had found that the NLR and PLR were independently predictive factors for poorer survival [31]. However, in other two retrospective analysis of the same year from Xiao-li Wei et al and Xiao-ling $\mathrm{Xu}$ et al (the number of enrolled cases all more than 400), this tendency was not observed in ESCC [20, 21]. Similarly, the preoperative NLR and PLR also lacked the prognostic ability for DFS or OS in patients with stage T1N0 ESCC in our study ( $p=$ 0.235 and 0.126 , respectively), but which was completely inconsistent with Xuan Xie's study in early stage ESCC (only 43 patients) [32]. The inconsistent findings in present studies may be attributed to following reasons:

Firstly, to these patients with early stage tumors, host may withstand a mild inflammatory burden leading to overall low NLR and PLR. For instance, the best cut-off values of NLR (1.976) and PLR (103.2) in our stage T1N0 ESCC patients were smaller than the previous EC cohort with non-selected stages (range of NLR cut-off: 2.0-5.0, range of PLR cut-off: 103.0-163.8) $[20,21,31]$. Secondly, up to date, the best cut-off values of these ratios were varies in published reports, which may weaken the clinical application and prognostic ability in EC with different stages, length of tumor, depth of tumor invasion, and lymph node 
metastasis etc $[19,31]$. In the meta-analysis conducted by Paramanathan A et al, the NLR cut-off of 5 showed significantly poorer survival with less heterogeneity in solid tumor [33]. Obviously, the optional NLR cut-off with less heterogeneity in ESCC has yet to be evaluated well, which need to verified in multicenter, lager cohort studies. Thirdly, a lot of inflammation-related disease, such as non-alcoholic fatty liver disease, metabolic syndrome, essential hypertension, infection etc, and premedication may affect the level of NLR and PLR [34, 35]. Fifthly, the time interval of these patients who were enrolled in this study was from January 2005 to December 2012 (8 years). During this long period, much progress has been made in peripheral blood cells count, thus the selection bias may be unavoidable. [36]. The last four were also the limitations of this retrospective analysis.

A simultaneously combination of CRP and white cell count in the prognostic index (PI), which is another readily available and well standardized prognostic scores like with (m/HS-m)GPS, has been confirmed as predictive marker for survival in lung cancer and colorectal cancer [37, 38]. However, no study is available regarding the prognostic role of PI in ESCC up to now and we showed for the first time. Even though the AUC values of the PI $(0.650,95 \% \mathrm{CI}$ : $0.557-0.744, p=0.004$, Fig. 2) was higher than the other inflammation-based prognostic scores in this study, but it was not significantly predicative value for RFS or OS in ESCC (or at least in stage T1N0) by multivariate analysis. Thus, the negative findings will prompt us to explore the prognostic value of PI in a larger prospective cohort with different stages ESCC.

In conclusion, our study determined the preoperative GPS and CAR as simple, inexpensive, readily available predictor for long-term survival in stage T1N0 ESCC patients who underwent esophagectomy. To these patients with high GPS or elevated CAR, endoscopic resection (ER) may not suitable and postoperative adjuvant chemotherapy may be considered.

\section{Supplementary Material}

Supplementary tables.

http://www.jcancer.org/v09p0807s1.pdf

\section{Acknowledgements}

We should thank the department of follow-up for recording recurrence and death data in detail. Besides, we are supposed to express our gratitude for the support and suggestion in this study from staffs at department of thoracic surgery.

\section{Competing Interests}

The authors have no conflicts of interest to disclose.

\section{References}

1. Chen W, Zheng R, Baade PD, Zhang S, Zeng H, Bray F, et al. Cancer statistics in China, 2015. CA Cancer J Clin. 2016; 66: 115-32.

2. Arnold M, Soerjomataram I, Ferlay J, Forman D. Global incidence of oesophageal cancer by histological subtype in 2012. Gut. 2015; 64: 381-7.

3. Chen MF, Yang YH, Lai CH, Chen PC, Chen WC. Outcome of patients with esophageal cancer: a nationwide analysis. Annals of surgical oncology. 2013; 20: 3023-30.

4. Rustgi AK, El-Serag HB. Esophageal carcinoma. The New England journal of medicine. 2014; 371: 2499-509.

5. Enzinger PC, Mayer RJ. Esophageal cancer. The New England journal of medicine. 2003; 349: 2241-52.

6. Song Z, Wang J, Lin B, Zhang Y. Analysis of the tumor length and other prognosis factors in pT1-2 node-negative esophageal squamous cell carcinoma in a Chinese population. World journal of surgical oncology. 2012; 10: 273.

7. Tanaka T, Matono S, Mori N, Shirouzu K, Fujita H. T1 squamous cell carcinoma of the esophagus: long-term outcomes and prognostic factors after esophagectomy. Annals of surgical oncology. 2014; 21: 932-8.

8. Teramoto H, Koike M, Tanaka C, Yamada S, Nakayama G, Fujii T, et al. Tumor budding as a useful prognostic marker in T1-stage squamous cell carcinoma of the esophagus. Journal of surgical oncology. 2013; 108: 42-6.

9. Holscher AH, Bollschweiler E, Schroder W, Metzger R, Gutschow C, Drebber U. Prognostic impact of upper, middle, and lower third mucosal or submucosal infiltration in early esophageal cancer. Annals of surgery. 2011; 254: 802-7; discussion 7-8.

10. Teoh AY, Chiu PW, Wong SK, Ng EK. Multifocal neoplasia and nodal metastases in $\mathrm{T} 1$ esophageal carcinoma: implications for endoscopic treatment. Annals of surgery. 2010; 251: 186-7.

11. Osugi J, Muto S, Matsumura Y, Higuchi M, Suzuki H, Gotoh M. Prognostic impact of the high-sensitivity modified Glasgow prognostic score in patients with resectable non-small cell lung cancer. Journal of cancer research and therapeutics. 2016; 12: 945-51.

12. Grivennikov SI, Greten FR, Karin M. Immunity, inflammation, and cancer. Cell. 2010; 140: 883-99.

13. Ishizuka $M$, Nagata $H$, Takagi $K$, Horie $T$, Kubota $K$. Inflammation-based prognostic score is a novel predictor of postoperative outcome in patients with colorectal cancer. Annals of surgery. 2007; 246: 1047-51.

14. Templeton AJ, McNamara MG, Seruga B, Vera-Badillo FE, Aneja P, Ocana A, et al. Prognostic role of neutrophil-to-lymphocyte ratio in solid tumors: a systematic review and meta-analysis. Journal of the National Cancer Institute. 2014; 106: dju124.

15. McMillan DC. The systemic inflammation-based Glasgow Prognostic Score: a decade of experience in patients with cancer. Cancer treatment reviews. 2013; 39: 534-40.

16. Leung EY, Scott HR, McMillan DC. Clinical utility of the pretreatment glasgow prognostic score in patients with advanced inoperable non-small cell lung cancer. Journal of thoracic oncology : official publication of the International Association for the Study of Lung Cancer. 2012; 7: 655-62.

17. Song ZB, Lin BC, Li B, He CX, Zhang BB, Shao L, et al. Preoperative elevation of serum $C$-reactive protein as an indicator of poor prognosis for early-stage esophageal squamous cell carcinoma. The Kaohsiung journal of medical sciences. 2013; 29: 662-6.

18. Lis CG, Gupta D, Lammersfeld CA, Markman M, Vashi PG. Role of nutritional status in predicting quality of life outcomes in cancer--a systematic review of the epidemiological literature. Nutrition journal. 2012; 11: 27.

19. Hirahara N, Matsubara T, Hayashi $H$, Takai K, Fujii $Y$, Tajima $Y$, Impact of inflammation-based prognostic score on survival after curative thoracoscopic esophagectomy for esophageal cancer. Eur J Surg Oncol. 2015; 41: 1308-15.

20. Wei XL, Wang FH, Zhang DS, Qiu MZ, Ren C, Jin Y, et al. A novel inflammation-based prognostic score in esophageal squamous cell carcinoma: the C-reactive protein/albumin ratio. BMC cancer. 2015; 15: 350.

21. $\mathrm{Xu} \mathrm{XL}, \mathrm{Yu} \mathrm{HQ}, \mathrm{Hu} W$, Song $\mathrm{Q}$, Mao WM. A Novel Inflammation-Based Prognostic Score, the C-Reactive Protein/Albumin Ratio Predicts the Prognosis of Patients with Operable Esophageal Squamous Cell Carcinoma. PLoS One. 2015; 10: e0138657.

22. Jomrich G, Paireder M, Gleiss A, Kristo I, Harpain L, Schoppmann SF. Comparison of Inflammation-Based Prognostic Scores in a Cohort of Patients with Resectable Esophageal Cancer. Gastroenterology research and practice. 2017; 2017: 1678584 .

23. McMillan DC, Crozier JE, Canna K, Angerson WJ, McArdle CS. Evaluation of an inflammation-based prognostic score (GPS) in patients undergoing resection for colon and rectal cancer. International journal of colorectal disease. 2007; 22: 881-6.

24. Tian $\mathrm{R}$, Zhang F, Sun $\mathrm{P}, \mathrm{Wu}$ J, Yan $\mathrm{H}, \mathrm{Wu}$ AR, et al. The preoperative sensitive-modified Glasgow prognostic score is superior to the modified Glasgow prognostic score in predicting long-term survival for esophageal squamous cell carcinoma. Oncotarget. 2016; 7: 67485-94. 
25. Ma $Q$, Liu $W$, Jia $R$, Jiang $F$, Duan $H$, Lin $P$, et al. Inflammation-based prognostic system predicts postoperative survival of esophageal carcinoma patients with normal preoperative serum carcinoembryonic antigen and squamous cell carcinoma antigen levels. World journal of surgical oncology. 2016; $14: 141$.

26. Lindenmann J, Fink-Neuboeck N, Avian A, Pichler M, Habitzruther M, Maier A, et al. Preoperative Glasgow Prognostic Score as additional independent prognostic parameter for patients with esophageal cancer after curative esophagectomy. Eur J Surg Oncol. 2017; 43: 445-53.

27. Okuno T, Wakabayashi M, Kato K, Shinoda M, Katayama H, Igaki H, et al. Esophageal stenosis and the Glasgow Prognostic Score as independent factors of poor prognosis for patients with locally advanced unresectable esophageal cancer treated with chemoradiotherapy (exploratory analysis of JCOG0303). International journal of clinical oncology. 2017.

28. Kinoshita A, Onoda H, Imai N, Iwaku A, Oishi M, Tanaka K, et al. The C-reactive protein/albumin ratio, a novel inflammation-based prognostic score, predicts outcomes in patients with hepatocellular carcinoma. Annals of surgical oncology. 2015; 22: 803-10.

29. Otowa Y, Nakamura T, Yamamoto M, Kanaji S, Matsuda Y, Matsuda T, et al. C-reactive protein to albumin ratio is a prognostic factor for patients with cStage II/III esophageal squamous cell cancer. Diseases of the esophagus : official journal of the International Society for Diseases of the Esophagus. 2017; 30: $1-5$.

30. Park HC, Kim MY, Kim CH. C-reactive protein/albumin ratio as prognostic score in oral squamous cell carcinoma. Journal of the Korean Association of Oral and Maxillofacial Surgeons. 2016; 42: 243-50.

31. Yodying H, Matsuda A, Miyashita M, Matsumoto S, Sakurazawa N, Yamada $\mathrm{M}$, et al. Prognostic Significance of Neutrophil-to-Lymphocyte Ratio and Platelet-to-Lymphocyte Ratio in Oncologic Outcomes of Esophageal Cancer: A Systematic Review and Meta-analysis. Annals of surgical oncology. 2016; 23: 646-54.

32. Xie X, Luo KJ, Hu Y, Wang JY, Chen J. Prognostic value of preoperative platelet-lymphocyte and neutrophil-lymphocyte ratio in patients undergoing surgery for esophageal squamous cell cancer. Diseases of the esophagus : official journal of the International Society for Diseases of the Esophagus. 2016; 29: 79-85.

33. Paramanathan A, Saxena A, Morris DL. A systematic review and meta-analysis on the impact of pre-operative neutrophil lymphocyte ratio on long term outcomes after curative intent resection of solid tumours. Surgical oncology. 2014; 23: 31-9.

34. Alkhouri N, Morris-Stiff G, Campbell C, Lopez R, Tamimi TA, Yerian L, et al. Neutrophil to lymphocyte ratio: a new marker for predicting steatohepatitis and fibrosis in patients with nonalcoholic fatty liver disease. Liver international : official journal of the International Association for the Study of the Liver. 2012; 32: 297-302.

35. Balta S, Cakar M, Demirkol S, Arslan Z, Akhan M. Higher neutrophil to lymhocyte ratio in patients with metabolic syndrome. Clinical and applied thrombosis/hemostasis : official journal of the International Academy of Clinical and Applied Thrombosis/Hemostasis. 2013; 19: 579.

36. Beaufrere H, Ammersbach M, Tully TN, Jr. Complete blood cell count in psittaciformes by using high-throughput image cytometry: a pilot study. Journal of avian medicine and surgery. 2013; 27: 211-7.

37. Proctor MJ, Morrison DS, Talwar D, Balmer SM, Fletcher CD, O'Reilly DS, et al. A comparison of inflammation-based prognostic scores in patients with cancer. A Glasgow Inflammation Outcome Study. European journal of cancer (Oxford, England : 1990). 2011; 47: 2633-41.

38. Kasymjanova G, MacDonald N, Agulnik JS, Cohen V, Pepe C, Kreisman H, et al. The predictive value of pre-treatment inflammatory markers in advanced non-small-cell lung cancer. Current oncology (Toronto, Ont). 2010; 17: 52-8.

39. Liu J.S., et al. A nomogram to predict prognostic values of various inflammatory biomarkers in patients with esophageal squamous cell carcinoma. Am J Cancer Res, 2015. 5(7): 2180-9.

40. Henry M.A., et al. The Glasgow Prognostic Score. An useful tool to predict survival in patients with advanced esophageal squamous cell carcinoma. Acta Cir Bras, 2015. 30(8): 580-5.

41. Ohira M., et al. Glasgow Prognostic Score as a Prognostic Clinical Marker in T4 Esophageal Squamous Cell Carcinoma. Anticancer Res, 2015. 35(9): 4897-901.

42. Kimura J. et al., Evaluation of the Glasgow Prognostic Score in patients receiving chemoradiotherapy for stage III and IV esophageal cancer. Dis Esophagus, 2016. 29(8): 1071-1080.

43. Toyokawa T., et al. The pretreatment Controlling Nutritional Status (CONUT) score is an independent prognostic factor in patients with resectable thoracic esophageal squamous cell carcinoma: results from a retrospective study. BMC Cancer, 2016. 16: 722

44. Ikeguchi M., et al. Evaluation of prognostic markers for patients with curatively resected thoracic esophageal squamous cell carcinomas. Mol Clin Oncol, 2016. 5(6): 767-772. 